\title{
Therapeutic effect of Mora combined with vasodilators for sudden hearing loss
}

\begin{abstract}
Objective To investigate the therapeutic effect of Mora combined with vasodilators for sudden hearing loss.

Methods: 120 patients (128 ears) suffering from sudden hearing loss and hospitalized in our department from Oct 2013 to Oct 2016 were included. According to the admission time, all the patients were randomly divided into two groups. 60 cases (66 ears) in group A received treatment of Mora combined with vasodilators, glucocorticoids and neurotrophic drugs. 60 cases ( 62 ears) in group B were treated by using vasodilators, glucocorticoids and neurotrophic drugs. The treatment course was 12 days for both groups. Their therapeutic effects were observed and compared.

Results: The total effective rate of group A was $90.91 \%$, which was higher than that of group B (74.19\%). The difference of total effective rate between both groups was statistically significant $(\mathrm{P}<0.05)$

Conclusion: Mora combined with vasodilators can significantly improve the therapeutic effect without any side effect which is worth applying and recommending in the treatment of sudden hearing loss.
\end{abstract}

Keywords: sudden hearing loss, mora, biotherapy, combined treatment

\author{
Volume II Issue 2 - 2018
}

Zhao Dong, Dai Lin-lin, Xu Pin, Zhou Bin, Wang Chun-ying

Department of Otolaryngology, the Forth Hospital Affiliated to Harbin Medical University, China

Correspondence: Zhao Dong, Department of Otolaryngology, the Forth Hospital Affiliated to Harbin Medical University, Harbin I50000, China, Email zhao_dong_mail@|26.com

Received: February 23, 2018 | Published: March II, 2018

\section{Introduction}

Sudden hearing loss (SHL), also called idiopathic sudden sorineural hearing loss (ISSHL), refers to sudden neural hearing loss accompanied possibly with tinnitus, dizziness, aural fullness and other clinical symptoms, caused by uncertain reasons within 3 days. SHL is a common emergency for otolaryngology and its morbidity is on the rise in recent years. ${ }^{1}$ Causes and pathogenesis of SHL are complicated and unclear, which could be connected with the microcirculation malfunction of inner ears, Ménière disease, viral infection, immunity and vascular disease. ${ }^{2}$ Even though, progress has been made to a certain extent in the treatment and efficacy evaluation of SHL, there is no clear standard, and most of the treatments perform poorly in prognosis. ${ }^{3}$ This research mainly discusses the therapeutic effect of Mora combined with vasodilators for SHL.

\section{Data \& method}

\section{General data}

120 SHL patients (128 ears) treated in our department from October 2013 to October 2016 were selected as subjects for the research. All patients received CT scans of the temporal bone and MRI examinations of the internal auditory canal, and had no organic diseases. Concurrently, patients with past history of drug allergies or diabetes, who are unable to use the combined treatment of glucocorticoid, and those with major organic failures were excluded from the research. Patients were numbered and marked according to their visitation date and were randomly divided into two groups by their marked numbers (odd or even). 60 cases (66 ears) in group A received treatment of Mora combined with vasodilators, glucocorticoids and neurotrophic drugs. 60 cases (62 ears) in group B were treated with the combined use of vasodilators, glucocorticoids and neurotrophic drugs. General conditions of patients during admission were not statistically different $(\mathrm{P}>0.05)$ in Table 1.

Table I Comparison of General Conditions (Case $\bar{x} \pm s$ )

\begin{tabular}{lllll}
\hline Clinic Data & Group A & Group B & t & P \\
\hline Number of Cases & 60 & 60 & - & - \\
Number of Ears & 66 & 62 & - & - \\
Gender & & & & \\
Male & 28 & 26 & 2.1 & 0.112 \\
Female & 32 & 34 & 1.4 & 0.139 \\
Period (d) & & & & \\
$\leq 3$ & 16 & 28 & 1.6 & 0.145 \\
$4 \sim 7$ & 24 & 16 & 1.2 & 0.223 \\
$>7$ & 20 & 16 & 3.6 & 0.439 \\
Age (years) & & & & \\
$<30$ & 10 & 12 & 3.1 & 0.516 \\
$30 \sim 60$ & 22 & 24 & 2.1 & 0.241 \\
$>60$ & 28 & 24 & 1.7 & 0.158 \\
SHL Type & & & & \\
HF Down & 22 & 20 & 2.9 & 0.154 \\
LF Down & 10 & 14 & 2.2 & 0.562 \\
Plain Down & 14 & 12 & 2.8 & 1.178 \\
THL & 14 & 14 & 2.1 & 0.397 \\
Complication & & & & \\
Dizziness & 30 & 22 & 2.9 & 0.090 \\
Tinnitus & 50 & 44 & 1.5 & 0.110 \\
\hline
\end{tabular}

\section{Treatment}

All patients received combined treatment with regular care and 
psychological counseling, with 12 days as one treatment course Drug \& usage: Jinnaduo (Extract of Ginkgo Biloba Leaves Tablets) $25 \mathrm{mg}+$ normal saline $250 \mathrm{ml}$ intravenous injection, once a day for 12 days; hexadecadrol $10 \mathrm{mg} / \mathrm{d}$ for three days, then $5 \mathrm{mg} / \mathrm{d}$ till the $10^{\text {th }}$ day; 52 patients with dizziness were treated with betahistine tablets (Minshilang, 6mg/tablet), 1 tablet is orally taken three times a day. Group A were added with the use of biological therapy of Mora for once every day, $40 \mathrm{~min}$ on each occasion, with 12 days as one treatment course. All patients in Group A and Group B received pure tone audiometry every 4 days during treatment. All treatments lasted for only one treatment course.

\section{Evaluation of effects}

Treatment efficacy for hearing loss was evaluated according to the 2015 Guide for Diagnosis and Treatment of SHL,${ }^{4}$ which includes $\circ 11$ Cured: damaged hearing ability is restored to normal or reach normal level, or restored to its previous level; $\circ 22$ Effectual: average damaged

Table 2 Comparison of Therapeutic Effect (Ears, \%) hearing ability is improved by more than $30 \mathrm{~dB}$; 033 Effective: damaged hearing ability is improved by 15 to $30 \mathrm{~dB}$; $\circ 44$ Ineffective: damaged hearing ability is improved by less than $15 \mathrm{~dB}$ or declines. Total efficiency $=($ cured ears + effectual ears + effective ears $) /$ total ears $* 100 \%$.

\section{Statistical processing}

Data was gathered and processed using statistical software SPSS 17.0; measurement data was shown as $\bar{x} \pm s$; inter-group comparison is tested by $t$ and inter-group rate $x^{2}$; difference is statistically significant only when $P<0.05$.

\section{Results}

After one treatment course, the total effective rate of Group A was $90.91 \%$, higher than that of Group B which was $74.19 \%$. The difference was statistically significant $(=0.02)$ in Table 2 . No side effects were observed during treatment in both groups.

\begin{tabular}{llllllll}
\hline Group & Ears & Cured & Effectual & Effective & Ineffective & $\begin{array}{l}\text { Total } \\
\text { effective rate }\end{array}$ \\
\hline $\begin{array}{l}\text { Group } \\
\text { A }\end{array}$ & 66 & 28 & 18 & 14 & 6 & $90.91^{*}$ \\
$\begin{array}{l}\text { Group } \\
\text { B }\end{array}$ & 62 & 18 & 12 & 16 & 16 & 74.19
\end{tabular}

Note: $* P=0.02$, compared with Group B

\section{Discussion}

Clinical treatment for SHL mainly uses glucocorticoids, vasodilators, anticoagulants and HPO. It has been found that glucocorticoids could trigger stress reaction with certain hormone receptors in the cytoplasm, which further alleviates SHL. Such hormone receptors ${ }^{5,6}$ widely exist in vestibule and cochlea, and mostly concentrate in the spiral ligament. Glucocorticoids and relevant receptors generate hormone-receptor compound to enter the cell nucleus. By increasing the transcription of anti-inflammatory genes and decreasing that of the inflammatory genes, it alleviates inflammation and further prevents other related illness. Therefore, glucocorticoids combined with vasodilators are mainly used to treat SHL. ${ }^{7-9}$

Treatment in combination with HPO or anticoagulants was proven effective for some patients. ${ }^{10,11}$ But not all SHL patients are suitable to undergo the treatment of glucocorticoids, especially for the aged as it often triggers other systematic diseases. Also, elder patients are more sensitive to the side effects of hormone and hormone on its own cannot be used for a long period of time. ${ }^{12-15}$ Patients in this research all received hormone treatment for one course and reported no side effects. Clinical researchers found that TCM is more suitable for treating SHL to some extent. ${ }^{16}$ Mora treatment is based on and developed from quantum physics, electromagnetic oscillation theory, bio-electronic environment theory, TCM channel and collateral channels \& acupuncture theory and natural homeopathy.

As is known to all, the human body is composed of millions of cells. Similar cells gather together to form human organs and different organs collaborate to ensure the regular running and health of the body. Also, each cell has its own magnetic field and only when they combine and collaborate can the body form a regular bio-magnetic field. When the body gets ill or certain organs fail, such bio-magnetic field would change and when the illness gets worse, corresponding symptoms would appear in the body. Mora treatment mainly adopts the technology of resonance of biological electromagnetic waves and is developed based on the theory that biologic cells have biological electromagnetic field effect and the material has wave-particle duality. Mora separates and analyzes irregular bio-magnetic fields of human body, and uses the electromagnetic wave system of the body to treat diseases. It could filter the electromagnetic waves of the human body, separate them into healthy ones and pathologic ones and use the former to balance the latter to achieve balance of the system and help the body to heal itself.

During Mora treatment, patients only need to sit with their hands separated and holding positive and negative electrode bars with their feet bared on the pedal, and Mora operators would then proceed with the rest of the treatment. There is no taking of any injection or drug. Mora, as a noninvasive treatment, could be used as a vital means to treat SHL for the time being. This research finds that the therapeutic effect of Mora combined with vasodilators for treating SHL could be $90.91 \%$, significantly higher than that of simply using vasodilators combined with glucocorticoids at $74.19 \%$, indicating that the former treatment outperforms the latter.

\section{Conclusion}

As a noninvasive treatment, Mora adjusts the state of body to actively prevent invasion of illness and could be a vital means for treating SHL. To sum up, Mora combined with vasodilators can significantly improve the therapeutic effect which is worth applying and recommending in the treatment of SHL. 


\section{Acknowledgments}

None

\section{Conflict of interest}

Author declares there is no conflict of interest towards this manuscript.

\section{References}

1. Zhang $\mathrm{X}, \mathrm{Xu} \mathrm{X}, \mathrm{Ma} \mathrm{W}$, et al. A clinical study of sudden deafness Acta Otolaryngol. 2015;135(10):1030-1035.

2. Nomura Y. Sudden Deafness. Morphological Aspects of Inner Ear Disease. 2014;90(12):85-99.

3. Lu Xinmin. Hormone Application in the Early Treatment of Sudden Deafness and its Curative Effect Evaluation Report. Chinese and Foreign Medicine. 2016;3(9):14-18.

4. Otolaryngology Department Magazine Editorial Committee of Chinese Medical Association, Chinese Medical Association Otolaryngology Head and Neck Surgery Branch. Sudden Deafness Diagnosis and Treatment Guidance (2015). Chinese Journal of Otolaryngology Head and Neck Surgery. 2015;50(6):443-447.

5. Mingming W, Zhaomin F, Zhiqiang H, et al. Effect of Glucocorticoid Local Injection and General Application in Idiopathic Sudden Deafness Classification Treatment. Chinese Journal of Otolaryngology Head and Neck Surgery. 2014;49(1):11-15.

6. Ping C, Jinhui Z. Curative Effect Analysis for Related Factors Influencing the Tinnitus Patients with Sudden Deafness. Audiology and Speech Diseases Journa. 2013;21(1):37-40.

7. Xiaodong H. Treatment Effect Observation of Alprostadil for Sudden Deafness. Medical Forum Journal. 2014;5(7):29-31.

8. Faxiang F, Lei S. Clinical Research on Injection of Dexamethasone in the Ear Drum for Treating Diabetes with Sudden Deafness. Chinese Otolaryngology Skull Base Surgery Journal. 2012;19(1):38-40.
9. Shijun L, Zhen S, Xiaobing Z. The Curative Effect of Steroid Hormones Feeding in the Ears on Sudden Deafness. Chinese Otolaryngology Skull Base Surgery Journal. 2012;18(4):325-327.

10. Renliang Q, Yingfeng L, Fuhua Z, et al. 22 Clinical Observations of Comprehensive Hyperbaric Oxygen Treatment for Diabetic. Chinese sailing medicine and Hyperbaric Oxygenation Medicine Journal. 2015;22(2):153-154.

11. Yuanfu H, Mei X, Jintian O, et al. Clinical Effect of Alprostadil and Early Hyperbaric Oxygen for Treating Sudden Deafness Tinnitus. Contemporary Chinese Medicine. 2014;21(21):109-111.

12. Hongxia G, Zhijian Z, Yang J. Research Progress of Glucocorticoid Treatment of Sudden Deafness. Chinese Medicine Guide. 2016;5(4):S1-S35.

13. Cohen PA. Vinpocetine: An Unapproved Drug Sold as a Dietary Supplement. Mayo Clinic Proceedings. 2015;90(10):1455.

14. Jiahui W, Guangming L, Yizan W, et al. 30 Cases of Clinical Observation about Glucocorticoid Treating Severe Chronic Obstructive Pulmonary Disease and Diabetes. Chinese National Folk Medicine. 2016;25(4):88-89.

15. Luyun J, Hui X, Jue X, et al. 42 Cases About Injecting Budesonide suspension Liquid to Ear Drum to Treat Sudden Deafness of Combined Type 2 Diabetes. Shandong University Ent Eye Journal. 2015;29(3):20-23.

16. Guohua D, Dengxiao R. Clinical Curative Effect Observation of Combining Chinese and Western Medicine for the Sudden Deafness Later Treatment. China Metallurgical Industry Medical Journal. 2014;31(4):467-468. 